\title{
ON INTEGRALITY AND GOING-DOWN INSIDE THE FIXED RING OF A MONOID RING
}

\author{
David E. Dobbs and Jay Shapiro \\ Received: 6 July 2015; Revised 12 September 2015 \\ Communicated by Sarah Glaz
}

\begin{abstract}
An example is given of a finitely generated abelian torsion-free monoid $S$ on which the group $G$ with two elements acts via semigroup automorphisms such that for any field $K$, when the given action is extended so that $G$ acts on the monoid ring $K[X ; S]$ via ring automorphisms that fix $K$ elementwise, the ring extension $K\left[X ; S^{G}\right] \subseteq(K[X ; S])^{G}$ is not integral and does not satisfy the going-down property.
\end{abstract}

Mathematics Subject Classification (2010): 13A50, 13B21, 13A15

Keywords: Commutative ring, ring extension, group action, fixed ring, integrality, going-down, semigroup, fixed semigroup, semigroup ring, INC, LO, factor semigroup, maximal ideal, Krull dimension, orbit, locally finite

\section{Introduction}

All rings considered below are commutative with 1; all semigroups are abelian and written additively. All actions of a group on a ring (resp., on a semigroup) are assumed to be via ring automorphisms (resp., semigroup automorphisms). If a group $G$ acts on a ring $A$ (resp., on a semigroup $S$ ), then $A^{G}:=\{a \in A \mid g \cdot a=a$ for all $g \in G\}$ (resp., $S^{G}:=\{s \in S \mid g \cdot s=s$ for all $g \in G\}$ ), the fixed ring (resp., fixed semigroup) of the given action. Recall (cf. [8]) that an action of a group $G$ (on either a semigroup $S$ or a ring $A$ ) is said to be locally finite if each $G$-orbit (of $S$ or $A$, as the case may be) is finite. Following [9, page 28], we let GD, INC, and LO respectively denote the properties of going-down, incomparability, and lying-over for ring extensions.

In this paragraph and the subsequent two paragraphs, we suppose that a group $G$ acts on a ring $A$. A well known result (cf. [1, Exercise 12, page 68]) states that if $G$ is finite, then the ring extension $A^{G} \subseteq A$ is integral. It was noted by Northcott [10] that the usual proof of this result actually yields the same conclusion if one assumes only that the action of $G$ on $A$ is locally finite. (Northcott's terminology for "locally finite" was "of finite character.") Then, still assuming that the action of 
$G$ on $A$ is locally finite, Northcott went on to show in [10, Theorem 6] that $A^{G} \subseteq A$ satisfies GD; and then [10, Theorem 8] established the more general fact that (if the action is locally finite, then) the canonical map $\operatorname{Spec}(R) \rightarrow \operatorname{Spec}\left(R^{G}\right)$ is open in the Zariski topology.

Unaware of the existence of [10], the authors later gave a ring-theoretic proof in [2, Corollary 2.16] that if $G$ is finite, then $A^{G} \subseteq A$ satisfies universally GD (in the sense that $A^{G}\left[\left\{X_{i}\right\}\right] \subseteq A\left[\left\{X_{i}\right\}\right]$ satisfies GD for each finite set $\left\{X_{i}\right\}$ of algebraically independent indeterminates over $A$ ), and in particular, $A^{G} \subseteq A$ satisfies GD. Later, we gave a ring-theoretic proof in [3, Theorem 2.2] that if $G$ is locally finite (on $A$ ), then $A^{G} \subseteq A$ is an integral extension that satisfies universally GD. Nevertheless, it is not always the case that $A^{G} \subseteq A$ satisfies GD. Indeed, in [4, Theorem 3.5], we presented an example where $A^{G} \subseteq A$ does not satisfy GD, with $G(\cong \mathbb{Z})$ necessarily not being locally finite on $A$.

Much of [3] concerned the relationship between $A^{G} \subseteq A$ being an integral extension and $G$ being locally finite on $A$. In particular, [3, Proposition 2.6] showed that if $A$ is a (commutative integral) domain, then $A^{G} \subseteq A$ is integral (if and) only if $G$ is locally finite on $A$. However, the "only if" assertion can fail if $A$ is not a domain, as [3, Example 2.5] gave a Boolean ring $A$ and a denumerably generated group $G$ acting on $A$ such that $A^{G} \subseteq A$ is integral while $G$ is not locally finite on $A$. On the other hand, it is not always the case that $A^{G} \subseteq A$ is integral. Indeed, two different constructions for examples were given in [3, Example 2.14] where $A^{G} \subseteq A$ fails to satisfy the INC property (which, of course, does hold for all integral extensions). In the same vein, [3, Example 2.16] gave an example where $A$ is a Noetherian domain (such as a polynomial ring in finitely many variables over a PID), $G \cong \mathbb{Z}$ and $A^{G} \subseteq A$ fails to satisfy the LO property (which, of course, does hold for all integral extensions). In view of the above background, it seems reasonable to suspect that if a finite group $G$ acts on a ring $A$ that is "somewhat like" the polynomial ring in finitely many variables over a field, then any ring extension that is "closely related to" $A^{G} \subseteq A$ should be integral and satisfy GD.

How can one convert the above suspicion into a conjecture? We begin with the group $G$ with only two elements and an arbitrary field $K$. The ring $A$ is taken to be the semigroup ring $K[X ; S]$ over a suitably chosen finitely generated (abelian) torsion-free cancellative monoid $S$ that $G$ acts on. (The "torsion-free cancellative" condition ensures that the semigroup ring $K[X ; S]$ is a domain [7, Theorem 8.1]. As recalled below, the action of $G$ on $S$ extends to an action of $G$ on $K[X ; S]$ that fixes $K$ elementwise.) For the ring extension that is "closely related to" $A^{G} \subseteq A$ (that is, to $\left.(K[X ; S])^{G} \subseteq K[X ; S]\right)$, we take $K\left[X ; S^{G}\right] \subseteq(K[X ; S])^{G}$. The purpose 
of this article is to refute the above suspicion/conjecture by constructing a suitable $S$ and an action of $G$ on that $S$ so that $K\left[X ; S^{G}\right] \subseteq(K[X ; S])^{G}$ is not integral and does not satisfy GD.

We can proceed more generally, at least at first. Let $R$ be a ring, let $G$ be a group, and let $S$ be a semigroup that $G$ acts on (via semigroup automorphisms). Then this action can be extended (uniquely) to an action of $G$ on the semigroup ring $A:=R[X ; S]$ (via ring automorphisms) as follows: $g \cdot \sum_{i=1}^{n} r_{i} X^{s_{i}}=\sum_{i=1}^{n} r_{i} X^{g \cdot s_{i}}$ if $g \in G$, each $r_{i} \in R$ and each $s_{i} \in S$. It is easy to check that $R\left[X ; S^{G}\right] \subseteq$ $(R[X ; S])^{G}$ in general. In the brief Section 2, Proposition 2.1 (b) characterizes when $R\left[X ; S^{G}\right]=(R[X ; S])^{G}$. Such a result is desirable as it indicates which sorts of data $R, G, S$ should be avoided if one wishes to construct an example where $R\left[X ; S^{G}\right] \subseteq(R[X ; S])^{G}$ is not integral and does not satisfy GD. Then Sections 3 and 4 are devoted to the construction and verification of the desired example. To facilitate communication, we fix notation in those two sections. As permitted by Proposition 2.1 (b), we do fix $R$ as an arbitrary field $K$ and we do fix $G$ to be the group with only two elements. After defining the suitable monoid $S$, we devote the rest of Section 3 to proving the "is not integral" assertion. The (harder) proof of the "does not satisfy GD" assertion is carried out in Section 4, where we find it necessary to study the behavior of the semigroup ring (over $K$ ) of a suitable factor semigroup of $S$.

As usual, $\subset$ denotes proper inclusion and $|B|$ denotes the cardinal number of a set $B$. If a group $G$ acts on a semigroup $S$ and $s \in S$, we denote the $G$-orbit of $s$ by $\mathcal{O}_{G}(s)$; we will omit the subscript $G$ if there is no danger of confusion. Also, for an element $f$ of a semigroup ring $R[X ; S]$, the support of $f$, denoted by $\operatorname{supp}(f)$, is the (finite) set of elements $s \in S$ such that the coefficient of $X^{s}$ in $f$ is nonzero. For background facts and notation for semigroup rings, we follow [7]. For any unexplained ring-theoretic material, see [6], [9].

\section{When the semigroup ring of the fixed semigroup is the fixed ring}

In this brief section, we again consider the canonical action of a group on a semigroup ring that is induced by a given action of that group on the ambient semigroup. Proposition 2.1 (b) determines when the fixed ring is simply the semigroup ring of the fixed semigroup. This result will help us focus the construction in the later sections of a suitable group and semigroup ring such that the ring extension in question is neither integral nor going-down. 
Proposition 2.1. Let $G$ be a group and $S$ a semigroup such that $G$ acts on $S$ (via semigroup automorphisms). Let $R$ be a ring and let the given action be extended canonically so that $G$ acts on the semigroup ring $R[X ; S]$ (via ring automorphisms, with each element of $R$ being fixed by each element of $G)$. Then:

(a) For $f \in R[X ; S]$, the following conditions are equivalent:

(1) $f \in(R[X ; S])^{G}$;

(2) There exist finitely many finite $G$-orbits of $S$, say $A_{1}, \ldots, A_{n}$, and elements $r_{1}, \ldots, r_{n} \in R$ such that $f$ can be written in the form $f=$ $f_{1}+f_{2}+\cdots+f_{n}$ where each $f_{i}=r_{i}\left(\sum_{s \in A_{i}} X^{s}\right)$.

(b) $R\left[X ; S^{G}\right]=(R[X ; S])^{G}$ if and only if each $G$-orbit of $S$ is either infinite or a singleton set.

Proof. (a) (2) $\Rightarrow(1)$ Suppose (2). To prove (1), it suffices to show that if $g \in$ $G$, then $g \cdot f_{i}=f_{i}$ for each $i$. In fact, since $g$ permutes $A_{i}$, we have $g \cdot f_{i}=$ $r_{i}\left(\sum_{s \in A_{i}} X^{g \cdot s}\right)=r_{i}\left(\sum_{s \in A_{i}} X^{s}\right)=f_{i}$.

$(1) \Rightarrow(2)$ Suppose (1); that is, $f \in(R[X ; S])^{G}$. Without loss of generality, $f \neq 0$. Write $f=\sum r_{\zeta} X^{\zeta}$ (where, as usual, $r_{\zeta} \in R$ for all $\zeta \in S$ and only finitely many $r_{\zeta}$ are nonzero). We claim that if $s \in \operatorname{supp}(f)$, then $\mathcal{O}(s) \subseteq \operatorname{supp}(f)$. Indeed, since $f \in(R[X ; S])^{G}, f=\sum r_{\zeta} X^{\zeta}=\sum{ }_{\zeta} r_{\zeta} X^{g \cdot \zeta}$ for all $g \in G$. Thus, $\sum_{\zeta} r_{g^{-1} \cdot \zeta} X^{\zeta}=\sum_{t} r_{t} X^{g \cdot t}=f$ for all $g \in G$. It follows that if $\zeta \in S$, then $r_{\zeta}=r_{g^{-1} \zeta}$ for all $g \in G$, and so $r_{\zeta}=r_{h \cdot \zeta}$ for all $h \in G$. Hence, since $r_{s} \neq 0$, we have $r_{h \cdot s} \neq 0$ for all $h \in G$, which proves the above claim. In particular, if $s \in$ $\operatorname{supp}(f)$, then $\mathcal{O}(s)$ is finite.

Once again, let $s \in \operatorname{supp}(f)$. Write $\mathcal{O}(s)=\left\{s_{1}, \ldots, s_{m}\right\}$. Consider $h_{1}:=$ $r_{s_{1}} X^{s_{1}}+r_{s_{2}} X^{s_{2}}+\cdots+r_{s_{m}} X^{s_{m}}$ (recalling that each $r_{s_{i}} \in R$ ). As was shown above, $r_{s_{i}}=r_{s_{j}}$ for all $1 \leq i, j \leq m$. Then $h_{1}=r_{s} \sum_{t \in \mathcal{O}(s)} X^{t}$ is the desired summand $f_{1}$.

Now let $h_{2}:=f-f_{1}$. As in the proof that $(2) \Rightarrow(1)$, we see that $f_{1} \in(R[X ; S])^{G}$, and so $h_{2} \in(R[X ; S])^{G}$. Moreover, $s \notin \operatorname{supp}\left(h_{2}\right)$, and so $\operatorname{supp}\left(h_{2}\right) \subset \operatorname{supp}(f)$. Therefore, by strong induction on the cardinality of $\operatorname{supp}(f)$, there exist finitely many finite $G$-orbits of $S$, say $A_{2}, \ldots, A_{n}$, and elements $\rho_{2}, \ldots, \rho_{n} \in R$ such that $h_{2}$ can be written in the form $h_{2}=f_{2}+f_{2}+\cdots+f_{n}$ where $f_{i}=\rho_{i}\left(\sum_{s \in A_{i}} X^{s}\right)$ for each $i \geq 2$. Then, with $A_{1}:=\mathcal{O}(s), f=f_{1}+h_{2}$ can be written in the desired form.

(b) Recall that, in general, $R\left[X ; S^{G}\right] \subseteq(R[X ; S])^{G}$. Thus, our task is to prove that $(R[X ; S])^{G} \subseteq R\left[X ; S^{G}\right]$ if and only if each $G$-orbit of $S$ is either infinite or a singleton set. For the "if" assertion, suppose that each finite $G$-orbit of $S$ is a singleton set. Then, by (a), if $f \in(R[X ; S])^{G}$, it follows that there exist finitely many singleton-set $G$-orbits of $S$, say $A_{1}=\mathcal{O}\left(s_{1}\right), \ldots, A_{n}=\mathcal{O}\left(s_{n}\right)$, and elements 
$r_{1}, \ldots, r_{n} \in R$ such that $f=f_{1}+f_{2}+\cdots+f_{n}$ where each $f_{i}=r_{i}\left(\sum_{s \in A_{i}} X^{s}\right)=$ $r_{i} X^{s_{i}}$. In other words, $f=\sum r_{i} X^{s_{i}} \in R\left[X ; S^{G}\right]$, as desired. Finally, to prove the (contrapositive of) the "only if" assertion, it suffices to observe that if there exists $\zeta \in S$ such that $1<|\mathcal{O}(\zeta)|<\infty$, then $\sum_{t \in \mathcal{O}(\zeta)} X^{t}$ is an element of $(R[X ; S])^{G}$ that is not in $R\left[X ; S^{G}\right]$.

\section{Integrality and some running notation}

As riding notation for the rest of this article, we take $K$ to be an arbitrary field, $G=\{1, g\}$ to be a certain group with two elements, and $S$ to be a certain semigroup on which $G$ acts. After defining $g$ and $S$ and also identifying the elements of $S^{G}$, we will show in Proposition 3.2 that the ring extension $K\left[X ; S^{G}\right] \subseteq(K[X ; S])^{G}$ is not integral. (In the next section, it will be shown that this ring extension also fails to satisfy GD.) This is to be contrasted with the fact (which is a consequence of a well known result that was recalled in the Introduction) that the extension $(K[X ; S])^{G} \subseteq K[X ; S]$ is integral.

The desired semigroup $S$ will be constructed as a certain factor semigroup of the following free abelian monoid. Let $F$ be the free abelian monoid (written additively) on a set of five distinct symbols, say $\left\{A, D_{1}, D_{2}, D_{3}, D_{4}\right\}$. (One can view the elements of $F$ as being those elements of the corresponding free abelian group which, when viewed as integral linear combinations of $A, D_{1}, D_{2}, D_{3}, D_{4}$, have only nonnegative coefficients.) Consider the semigroup automorphism $g$ of $F$ that is determined by having $g$ fix $A$, interchange $D_{1}$ and $D_{2}$, and also interchange $D_{3}$ and $D_{4}$, with the definition of $g$ then extended linearly to the other elements of $F$. We next introduce (what turns out to be) a congruence relation $\equiv$ on the monoid $F$, as follows. If $x, y \in F$, then $x \equiv y$ if and only if either $x=y$ or we can write

$x=n A+n_{1} D_{1}+n_{2} D_{2}+n_{3} D_{3}+n_{4} D_{4}$ and $y=s A+s_{1} D_{1}+s_{2} D_{2}+s_{3} D_{3}+s_{4} D_{4}$

where $n=s>0$ and $\sum n_{i}=\sum s_{i}$. It is clear that $\equiv$ is an equivalence relation. It is also easy to check that $\equiv$ is a congruence relation on $F$. (In other words, if $u_{1} \equiv v_{1}$ and $u_{2} \equiv v_{2}$, then $u_{1}+u_{2} \equiv v_{1}+v_{2}$.) The desired semigroup $S$ is defined as the factor semigroup $S:=F / \equiv$. Note that $S$ is a(n abelian) monoid. It is also easy to check that if $u \equiv v$ in $R$, then $g(u) \equiv g(v)$. Consequently, the automorphism $g$ of $F$ induces a semigroup automorphism of $S$, which we also denote by $g$. As $g \circ g$ is the identity map, the group $G:=\{1, g\}$ has exactly two elements. (To verify the preceding assertion, one must check that $g: S \rightarrow S$ is not the identity map, but this is clear since $D_{1} \not \equiv D_{2}\left(=g\left(D_{1}\right)\right)$ in $F$.) 
Now that $S$ and $G$ have been defined, we next introduce some more riding notation. Let $a$ denote the $\equiv$-equivalence class represented by $A$ and, for $1 \leq i \leq$ 4 , let $d_{i}$ denote the $\equiv$-equivalence class represented by $D_{i}$. Also, if $s$ and $t$ are positive integers, let $b_{s, t}$ denote the $\equiv$-equivalence class represented by any element $s A+n_{1} D_{1}+n_{2} D_{2}+n_{3} D_{3}+n_{4} D_{4}$ where $\sum n_{i}=t$ (for instance, $s A+t D_{1}$ ).

The next result collects some useful information. Its proof is straightforward (via a somewhat tedious analysis of cases which ultimately depends on the definition of $\equiv$ ) and is left to the reader. Lemma 3.1 (a) will often see tacit use below, Lemma 3.1 (b) will be used at once, and Lemma 3.1 (c) will lead naturally to a set of identities listed in the proof of Proposition 4.1.

Lemma 3.1. (a) Each nonzero element of $S$ can be expressed in one of the following three ways: ma with $m>0 ; b_{s, t}$ with $s, t>0 ;$ and $\sum_{i=1}^{4} m_{i} d_{i}$ with each $m_{i} \geq 0$.

(b) The fixed semigroup $S^{G}$ is the set of elements

$\left\{m a, b_{s, t}, l\left(d_{1}+d_{2}\right)+k\left(d_{3}+d_{4}\right)\right\}$, where $m, l, k \geq 0$, and $s, t>0$.

(c) For positive integers $m, k, s, t$ and $n$, one has the following identities in $S^{G}: m a+k\left(d_{1}+d_{2}\right)=b_{m, 2 k}=m a+k\left(d_{3}+d_{4}\right), n b_{s, t}=b_{n s, n t}$, and $m a+b_{s, t}=b_{m+s, t}$.

Proposition 3.2. Let $K, S$ and $G$ be as above. Then $K\left[X ; S^{G}\right] \subset(K[X ; S])^{G}$ does not satisfy INC. In particular, this ring extension is not integral.

Proof. Since $\mathcal{O}_{G}\left(d_{1}\right)=\left\{d_{1}, d_{2}\right\}$ and $d_{1} \neq d_{2}$, it follows from Proposition 2.1 (b) that $K\left[X ; S^{G}\right] \subset(K[X ; S])^{G}$ is a proper inclusion. The "In particular" assertion follows from the fact that each integral extension satisfies INC (cf. [6, Corollary 11.2], [9, Theorem 44]). Next, observe that if $A \subseteq B \subseteq C$ are rings such that $B \subseteq C$ is an integral extension, then $A \subseteq B$ satisfies INC if and only if $A \subseteq C$ satisfies INC. (Indeed, the "only if" assertion follows because the union of a finite tower of INC extensions is an INC extension, while the "if" assertion follows easily from the fact that any integral extension satisfies the LO and goingup properties [9, Theorem 44].) We next apply this fact to the chain of rings $K\left[X ; S^{G}\right] \subseteq(K[X ; S])^{G} \subseteq K[X ; S]$. Note that since $G$ is finite, an above-mentioned result ensures that the extension $(K[X ; S])^{G} \subseteq K[X ; S]$ is integral. Hence, it suffices to show that $K\left[X ; S^{G}\right] \subseteq K[X ; S]$ does not satisfy INC.

Let $P$ denote the ideal of $K[X ; S]$ generated by set $\left\{X^{a}, X^{d_{1}}, X^{d_{3}}\right\}$. It is clear that $\left\{X^{d_{2}}+P, X^{d_{4}}+P\right\}$ generates the factor $\operatorname{ring} K[X ; S] / P$ as a $K$-algebra. 
Moreover, it is straightforward to check, using the definition of $\equiv$, that the subsemigroup of $S$ generated by the set $\left\{d_{2}, d_{4}\right\}$ is a free abelian monoid on (this set of) two elements. It follows easily (again by using the definition of $\equiv$ ) that $K[X ; S] / P$ is $K$-algebra isomorphic to the polynomial ring in two algebraically independent indeterminates over $K$. Therefore, $P$ is a prime ideal, but not a maximal ideal, of $K[X ; S]$. Pick a maximal ideal $M$ of $K[X ; S]$ that (properly) contains $P$ (for instance, let $M$ be the ideal of $K[X ; S]$ generated by $\left.\left\{X^{a}, X^{d_{1}}, X^{d_{2}}, X^{d_{3}}, X^{d_{4}}\right\}\right)$. Note that $Q:=P \cap K\left[X ; S^{G}\right]$ contains $X^{a}, X^{b_{s, t}}$ (for all positive integers $s$ and $t),\left(X^{d_{2}} \cdot X^{d_{1}}=\right) X^{d_{1}+d_{2}}$, and $\left(X^{d_{4}} \cdot X^{d_{3}}=\right) X^{d_{3}+d_{4}}$. Thus, by Lemma $3.1(\mathrm{~b})$, $Q$ contains $\left\{X^{s}: s \in S^{G}\right\}$. Consequently, $K\left[X ; S^{G}\right] / Q \cong K$, and so $Q$ is a maximal ideal of $K\left[X ; S^{G}\right]$. Therefore, $M \cap K\left[X ; S^{G}\right]$ must also be $Q$. As $P \subset M$, $K\left[X ; S^{G}\right] \subseteq K[X ; S]$ does not satisfy INC, to complete the proof.

Remark 3.3. Although one can easily check that the ambient semigroup $S$ inherits the "torsion-free" property from the additive structure of $\mathbb{Z}, S$ is not cancellative. (To see this, observe, for instance, that $a+d_{1}=a+d_{2}$ but $d_{1} \neq d_{2}$.) The semigroup $S^{G}$ also fails to be cancellative, as $a+d_{1}+d_{2}=a+d_{3}+d_{4}$ but $d_{1}+d_{2} \neq d_{3}+d_{4}$. Hence, by $\left[7\right.$, Theorem 8.1], neither $K[X ; S]$ nor its subring $K\left[X ; S^{G}\right]$ is a domain. In Section 4, it will be convenient to overcome the fact that $d_{1}+d_{2} \neq d_{3}+d_{4}$ by introducing a factor semigroup $H$ of $S^{G}$ which will turn out to be both cancellative and torsion-free.

\section{Going-down and more running notation}

The symbols $K, G$ and $S$ retain the fixed meanings assigned to them in the preceding section; the action of $G$ on $S$ and the induced action of $G$ on $K[X ; S]$ also remain as above. This section is devoted to proving that the extension $K\left[X ; S^{G}\right] \subset$ $(K[X ; S])^{G}$ does not satisfy GD. This proof will require us to define and study a suitable factor semigroup of $S^{G}$. That factor semigroup, denoted by $H$ below, will be defined via a suitable congruence relation on the monoid $S^{G}$.

The desired congruence relation on $S^{G}$ can be introduced as follows. Let $\sim$ be the smallest congruence relation on $S^{G}$ such that $k\left(d_{1}+d_{2}\right) \sim k\left(d_{3}+d_{4}\right)$ for any positive integer $k$. By using Lemma 3.1 (b) and the definitions of $\equiv$ and $\sim$, one easily checks that $\sim$ is well defined. Indeed, if $u$ and $v$ are distinct elements of $S^{G}$ with $u \neq v$, then $u \sim v$ if and only if $u$ and $v$ can be expressed as follows: $u=k\left(d_{1}+d_{2}\right)+l\left(d_{3}+d_{4}\right)$ and $v=k^{\prime}\left(d_{1}+d_{2}\right)+l^{\prime}\left(d_{3}+d_{4}\right)$ for some nonnegative integers $k, l, k^{\prime}$ and $l^{\prime}$ such that $k+l=k^{\prime}+l^{\prime}$ (and either $k \neq k^{\prime}$ or $l \neq l^{\prime}$ ). Let $H$ denote the factor semigroup $H:=S^{G} / \equiv$. Note that $H$ is a(n abelian) monoid. 
Now that $\sim$ and $H$ have been defined, we next denote the $\sim$-equivalence class of $a$ by $\alpha$, the $\sim$-equivalence class of $b_{s, t}$ by $\beta_{s, t}$ (for any positive integers $s$ and $t$ ), and the common $\sim$-equivalence class of $d_{1}+d_{2}$ and $d_{3}+d_{4}$ by $\delta$. From now on, the symbols $H, \alpha, \beta_{s, t}$ and $\delta$ are added to our list of running notation. It is important to observe that each nonzero element of $H$ can be expressed in exactly one of the following three ways (with the respective parameters $m ; s, t$; and $l$ being uniquely determined in every case): $m \alpha$ with $m>0 ; \beta_{s, t}$ (with $s>0$ and $t>0$ ); $l \delta$ with $l>0$.

The next result fulfills a promise that was made in Remark 3.3.

Proposition 4.1. The semigroup $H$ is cancellative and torsion-free.

Proof. It will be convenient to refer to a nonzero element of $H$ that is of the form $m \alpha, \beta_{s, t}$, or $l \delta$ as being of type 1 , type 2 , or type 3 , respectively. As noted above, each nonzero element of $H$ is of exactly one of these three types (with the associated parameters being uniquely determined). It will be useful to collect the following identities (where $m, l, n, s, t$ are arbitrary positive integers):

$$
\begin{aligned}
m \alpha+l \delta & =\beta_{m, 2 l} \\
n \beta_{s, t} & =\beta_{n s, n t} \\
\beta_{s, t}+l \delta & =\beta_{s, t+2 l} \text { and } \\
m \alpha+\beta_{s, t} & =\beta_{m+s, t} .
\end{aligned}
$$

We will show first that $H$ is torsion-free. This requires proving that if $h_{1}, h_{2} \in H$ and $n$ is a positive integer such that $n h_{1}=n h_{2}$, then $h_{1}=h_{2}$. The assertion is clear if $h_{1}$ or $h_{2}$ is 0 , and so we can assume that $h_{1}$ and $h_{2}$ are each nonzero. Note that if $0 \neq h \in H$, then $n h$ and $h$ are of the same type. Since $n h_{1}=n h_{2}$, it follows that $h_{1}$ and $h_{2}$ must be of the same type. In view of the uniqueness of parameters and the torsion-free property of the nonzero integers, the assertion follows easily in each of three cases (bearing in mind that $n(m \alpha)=(n m) \alpha, n \beta_{s, t}=\beta_{n s, n t}$, and $n(l \delta)=(n l) \delta)$.

It remains to show that $H$ is cancellative. This requires proving that if $e, f, h \in H$ are such that $h+e=h+f$, then $e=f$. Without loss of generality, $h \neq 0$. It follows easily from the above identities (together with evident facts such as $\beta_{s, t}+\beta_{s^{\prime}, t^{\prime}}=$ $\left.\beta_{s+s^{\prime}, t+t^{\prime}}\right)$ that $h+e \neq h$ if $e \neq 0$. Thus, without loss of generality, both $e$ and $f$ are nonzero. We now proceed with a somewhat tedious but straightforward analysis of three cases.

Case 1: $h$ is of type 3. Write $h=l \delta$ (with $l>0$ ). It follows from the second and third of the displayed identities that if one of $e$ or $f$ is also of type 3 , then both 
$e$ and $f$ are of type 3 , in which case one sees (by the cancellative property of the nonzero integers) that $e=f$. Similarly, if both $e$ and $f$ are of type 1 , then we can use the first of the displayed identities to conclude that $e=f$. Suppose next that one of $e$ and $f$ is of type 1 and the other is of type 2 , say with $e=m \alpha$ and $f=\beta_{s, t}$; then $\beta_{m, 2 l}=h+e=h+f=\beta_{s, t+2 l}$, whence $2 l=t+2 l$ and $t=0$, a contradiction. In the remaining subcase, both $e$ and $f$ are of type 2 ; write $e=\beta_{s, t}$ and $f=\beta_{s^{\prime}, t^{\prime}}$. Then $\beta_{s, t+2 l}=h+e=h+f=\beta_{s^{\prime}, t^{\prime}+2 l}$, whence $s=s^{\prime}$ and $t+2 l=t^{\prime}+2 l$, so $t=t^{\prime}$ and $e=f$, which completes the proof for Case 1 .

Case 2: $h$ is of type 1 . Write $h=m \alpha$ (with $m>0$ ). It follows from the first and fourth of the displayed identities that if one of $e$ or $f$ is also of type 1 , then both $e$ and $f$ are of type 1 , in which case one sees (by the cancellative property of the nonzero integers) that $e=f$. Similarly, if both $e$ and $f$ are of type 3 , then it follows easily from the first displayed identity that $e=f$. Suppose next that one of $e$ and $f$ is of type 2 and the other is of type 3 , say with $e=\beta_{s, t}$ and $f=k \delta$; then $\beta_{m+s, t}=h+e=h+f=\beta_{m, 2 k}$, whence $m+s=m$ (and $t=2 k$ ), so $s=0$, a contradiction. In the remaining subcase, both $e$ and $f$ are of type 2 ; write $e=\beta_{s, t}$ and $f=\beta_{s^{\prime}, t^{\prime}}$. Then $\beta_{m+s, t}=h+e=h+f=\beta_{m+s^{\prime}, t^{\prime}}$, whence $m+s=m+s^{\prime}$ and $t=t^{\prime}$, so $s=s^{\prime}$ and $e=f$, which completes the proof for Case 2 .

Case 3: $h$ is of type 2. Write $h=\beta_{s, t}$. If $e$ and $f$ are of the same type as each other, then an analysis of the three resulting subcases (that uses the above identities and elementary properties of the integers) easily leads to $e=f$. Three subcases remain. In the first of these, one of $e$ and $f$ is of type 1 and the other is of type 3 ; without loss of generality, write $e=m \alpha$ and $f=l \delta$. Then $\beta_{m+s, t}=$ $h+e=h+f=\beta_{s, t+2 l}$, whence $m+s=s$ (and $\left.t=t+2 l\right)$, so $m=0(=l)$, a contradiction. In the penultimate subcase, one of $e$ and $f$ is of type 1 and the other is of type 2 ; without loss of generality, write $e=m \alpha$ and $f=\beta_{s^{\prime}, t^{\prime}}$. Then $\beta_{m+s, t}=h+e=h+f=\beta_{s+s^{\prime}, t+t^{\prime}}$, whence $\left(m+s=s+s^{\prime}\right.$ and $) t=t+t^{\prime}$, so $t^{\prime}=0$, a contradiction. In the final subcase, one of $e$ and $f$ is of type 3 and the other is of type 2 ; without loss of generality, write $e=l \delta$ and $f=\beta_{s^{\prime}, t^{\prime}}$. Then $\beta_{s, t+2 l}=h+e=h+f=\beta_{s+s^{\prime}, t+t^{\prime}}$, whence $s=s+s^{\prime}$ (and $t+2 l=t+t^{\prime}$ ), so $s^{\prime}=0$, a contradiction. This completes the proof.

Corollary 4.2. Let $K, S, G$ and $H$ be as above. Then $\left(X^{d_{1}+d_{2}}-X^{d_{3}+d_{4}}\right) K\left[X ; S^{G}\right]$ is a prime ideal of $K\left[X ; S^{G}\right]$.

Proof. For each $p \in S^{G}$, let $[p]$ denote the $\sim$-equivalence class of $p$ in $H$. Then the assignment $X^{p} \mapsto X^{[p]}$ induces a (unique) surjective $K$-algebra homomorphism, say $f$, from the semigroup $\operatorname{ring} K\left[X ; S^{G}\right]$ (on)to the monoid $\operatorname{ring} K[X ; H]$. As we 
showed in Proposition 4.1 that $H$ is cancellative and torsion-free, it follows from [7, Theorem 8.1] that $K[X ; H]$ is an integral domain. Therefore, $\operatorname{ker}(f)$ is a prime ideal of $K\left[X ; S^{G}\right]$. It therefore suffices to prove that $\operatorname{ker}(f)$ is the ideal of $K\left[X ; S^{G}\right]$ that is generated by $X^{d_{1}+d_{2}}-X^{d_{3}+d_{4}}$.

Of course, $Y:=X^{d_{1}+d_{2}}-X^{d_{3}+d_{4}} \in \operatorname{ker}(f)$, because $d_{1}+d_{2} \sim d_{3}+d_{4}$. Now, it follows from the proof of the second assertion in [7, Corollary 7.3] that $\operatorname{ker}(f)$ is the set of all finite sums of elements of the form $r X^{u}-r X^{v}$ where $r \in K$ and $u \sim v$ in $S^{G}$. Thus, it suffices to prove that if $u \sim v$ in $S^{G}$ with $u \neq v$, then $Y$ divides $X^{u}-X^{v}$ in $K\left[X ; S^{G}\right]$. By the earlier description of the binary relation $\sim$, we can write $u=k\left(d_{1}+d_{2}\right)+l\left(d_{3}+d_{4}\right)$ and $v=k^{\prime}\left(d_{1}+d_{2}\right)+l^{\prime}\left(d_{3}+d_{4}\right)$ for some nonnegative integers $k, l, k^{\prime}$ and $l^{\prime}$ such that $N:=k+l=k^{\prime}+l^{\prime}$. Hence, $X^{u}-X^{v}$ can be written as

$$
X^{k\left(d_{1}+d_{2}\right)} \cdot\left\{X^{l\left(d_{3}+d_{4}\right)}-X^{l\left(d_{1}+d_{2}\right)}\right\}+\left\{X^{(k+l)\left(d_{1}+d_{2}\right)}-X^{k^{\prime}\left(d_{1}+d_{2}\right)+l^{\prime}\left(d_{3}+d_{4}\right)}\right\} .
$$

The first of the displayed summands is divisible by $Y$, since $X^{l\left(d_{3}+d_{4}\right)}-X^{l\left(d_{1}+d_{2}\right)}=$ $\left(X^{d_{3}+d_{4}}\right)^{l}-\left(X^{d_{1}+d_{2}}\right)^{l}$ is divisible by $X^{d_{3}+d_{4}}-X^{d_{1}+d_{2}}=-Y$ (for all nonnegative integers $l$ ). Hence, it suffices to prove that the second of the displayed summands is divisible by $Y$. Now, that second summand can be expressed as the sum $Z_{1}+Z_{2}$, where

$$
Z_{1}:=X^{(k+l)\left(d_{1}+d_{2}\right)}-X^{\left(k^{\prime}+l^{\prime}\right)\left(d_{3}+d_{4}\right)}=\left(X^{d_{1}+d_{2}}\right)^{k+l}-\left(X^{d_{3}+d_{4}}\right)^{k^{\prime}+l^{\prime}}=
$$

$\left(X^{d_{1}+d_{2}}\right)^{N}-\left(X^{d_{3}+d_{4}}\right)^{N}$ is divisible by $Y$; and

$Z_{2}=X^{\left(k^{\prime}+l^{\prime}\right)\left(d_{3}+d_{4}\right)}-X^{k^{\prime}\left(d_{1}+d_{2}\right)+l^{\prime}\left(d_{3}+d_{4}\right)}=X^{l^{\prime}\left(d_{3}+d_{4}\right)} \cdot\left\{X^{k^{\prime}\left(d_{3}+d_{4}\right)}-X^{k^{\prime}\left(d_{1}+d_{2}\right)}\right\}$,

which is also divisible by $Y$. It follows that $X^{u}-X^{v}$ is divisible by $Y$, which completes the proof.

We now complete the verification of the promised example.

Theorem 4.3. Let $K, S$ and $G$ be as above. Then the ring extension $K\left[X ; S^{G}\right] \subset$ $(K[X ; S])^{G}$ does not satisfy going-down.

Proof. Recall that Proposition 2.1 (b) ensures that $K\left[X ; S^{G}\right] \subset(K[X ; S])^{G}$ is a proper inclusion. Now, it is well known (and easy to prove) that if $A \subseteq B \subseteq C$ are rings such that both $A \subseteq B$ and $B \subseteq C$ satisfy GD, then $A \subseteq C$ also satisfies GD. We next apply this fact to the chain of rings $K\left[X ; S^{G}\right] \subseteq(K[X ; S])^{G} \subseteq K[X ; S]$. Note that since $G$ is finite, $(K[X ; S])^{G} \subseteq K[X ; S]$ does satisfy GD [3, Theorem 2.2] (cf. also [10], [2]). Therefore, it will suffice to show that $K\left[X ; S^{G}\right] \subset K[X ; S]$ does not satisfy GD. 
Consider the ring $A:=K\left[X_{1}, X_{2}, X_{3}, X_{4}\right]$, where the $X_{i}$ are commuting algebraically independent indeterminates over $K$, and the element $Y:=X_{1} X_{2}-X_{3} X_{4} \in$ $A$. It is well known that the $\operatorname{ring} B:=A / Y A$ is a domain, in fact, a Krull domain, as a consequence of [5, Proposition 14.5 (a)]. Since $A$ is a UFD, it follows that $Y$ is a prime element of $A$, and so $Y A$ is a prime ideal of $A$. Since $A$ is an affine domain (in fact, a Cohen-Macaulay ring), it is a (universally) catenarian domain of Krull dimension 4 (cf. [9, Theorem 151], [11, Corollaire 2, page III-24]); and the prime ideal $Y A$ of $A$ has height 1 (by, for instance, the Principal Ideal Theorem [9, Theorem 142]). It follows that the factor ring $B$ has Krull dimension 3. We proceed to find a useful ring that is isomorphic to $B$.

Consider the ideal $Q_{1}:=\left(X^{a}, X^{d_{1}+d_{2}}-X^{d_{3}+d_{4}}\right) K[X ; S]$ of $K[X ; S]$. We claim that $Q_{1}$ is a prime ideal of $K[X ; S]$. As in the proof of Proposition 3.2, it is straightforward to check, using the definition of $\equiv$, that the subsemigroup of $S$ generated by the set $\left\{d_{1}, d_{2}, d_{3}, d_{4}\right\}$ is a free abelian monoid on (this set of) four elements. It follows easily (again by using the definition of $\equiv$ ) that $K[X ; S] / X^{a} K[X ; S]$ is $K$-algebra isomorphic to $K\left[X_{1}, X_{2}, X_{3}, X_{4}\right](=A)$ via the $K$-algebra map induced by sending the coset represented by $X^{d_{i}}$ to $X_{i}$. Under this map, the image of $Q_{1} / X^{a} K[X ; S]$ is the ideal generated by $X_{1} X_{2}-X_{3} X_{4}$, that is, the ideal $Y A$. Consequently, standard homomorphism theorems yield $K$-algebra isomorphisms

$$
K[X ; S] / Q_{1} \cong\left(K[X ; S] / X^{a} K[X ; S]\right) /\left(Q_{1} / X^{a} K[X ; S]\right) \cong A / Y A=B,
$$

which we have seen is a domain. Therefore, $Q_{1}$ is a prime ideal of $K[X ; S]$, thus proving the above claim. The ideal $Q_{1}$ will be one of the three prime ideals that we will produce in data showing that $K\left[X ; S^{G}\right] \subset(K[X ; S])^{G}$ does not satisfy going-down.

By combining the second claim with Lemma 3.1 (b), $P_{1}:=Q_{1} \cap K\left[X ; S^{G}\right]$ is a prime ideal of $K\left[X ; S^{G}\right]$ which contains $\left(X^{a}, X^{d_{1}+d_{2}}-X^{d_{3}+d_{4}}\right) K\left[X ; S^{G}\right]$. Recall from Corollary 4.2 that $P_{2}:=\left(X^{d_{1}+d_{2}}-X^{d_{3}+d_{4}}\right) K\left[X ; S^{G}\right]$ is a prime ideal of $K\left[X ; S^{G}\right]$. Evidently, $P_{2} \subseteq P_{1}$. In fact, $P_{2} \subset P_{1}$, with $X^{a} \in P_{1} \backslash P_{2}$, since (to use notation from, and a result from the proof of, Corollary 4.2), $f\left(X^{a}\right)=X^{\alpha} \neq 0 \in$ $K[X ; H]$ ensures that $X^{a} \notin \operatorname{ker}(f)=P_{2}$. Thus (by the definition of the going-down property), it suffices to prove that there cannot exist a prime ideal $Q_{2}$ of $K[X ; S]$ which is contained in $Q_{1}$ and contracts to $P_{2}$.

Suppose, on the contrary, that such a prime ideal $Q_{2}$ does exist. Note first that $X^{d_{1}+d_{2}}-X^{d_{3}+d_{4}} \in P_{2} \subseteq Q_{2}$. Hence, if $Q_{2}$ also contained $X^{a}$, we would have $Q_{2}=Q_{1}$, a contradiction. On the other hand, we know that $X^{a}\left(X^{d_{i}}-X^{d_{j}}\right)=0$ for all $1 \leq i, j \leq 4$, since $a+d_{i}=b_{1,1}=d_{j}$. As $Q_{2}$ is a prime ideal of $K[X ; S]$ and $X^{a} \notin$ 
$Q_{2}$, it follows that $X^{d_{i}}-X^{d_{j}} \in Q_{2}$ for all $1 \leq i, j \leq 4$. Consequently, $K[X ; S] / Q_{2}=$ $K\left[X^{a}+Q_{2}, X^{d_{1}}+Q_{2}\right]$ is a $K$-algebra homomorphic image of a polynomial ring over $K$ in two (commuting algebraically independent) indeterminates. It follows that the Krull dimension of $K[X ; S] / Q_{2}$ is at most 2. On the other hand, since $Q_{2} \subseteq Q_{1}$, there is obviously a surjective $K$-algebra homomorphism from $K[X ; S] / Q_{2}$ onto $K[X ; S] / Q_{1}$. But the latter ring, as noted earlier, is $K$-algebra isomorphic to $B$, which we have seen has Krull dimension 3. We have obtained an absurdity, where there is a surjective ring homomorphism from a ring of Krull dimension at most 2 onto a ring of Krull dimension 3. This (desired) contradiction shows that no $Q_{2}$ can exist with the above properties. The proof is complete.

In closing, we give an example that illustrates that the above monoid $S$ needed to have a somewhat delicate construction in order to sustain the conclusions in Proposition 3.2 and Theorem 4.3. Remark 4.4 gives an example where we consider the above group $G$, a particular field $K$, and a new (to this article) finite monoid $T$ such that $K\left[X ; T^{G}\right] \subset(K[X ; T])^{G}$ is a proper ring extension which is integral and satisfies GD.

Remark 4.4. Let $G=\{1, g\}$ be the above group; specialize the ambient field to $K:=\mathbb{F}_{2}$, the field with two elements; and take the (additive abelian) monoid $T$ to be $T:=\{0, x, y\}$ in which every sum equals 0 . It is easy to check that an action of $G$ on $T$ (via semigroup automorphisms) can be defined by taking $g \cdot 0:=0$, $g \cdot x:=y$ and $g \cdot y:=x$ (with $1 \cdot z:=z$ for each $z \in T$ ). Note that $T^{G}=\{0\}$. Thus $K\left[X ; T^{G}\right]=K[X ;\{0\}]=K X^{0} \cong K$, and so $K\left[X ; T^{G}\right]$ is a field. (It will be convenient to identify this field with $K$.) It follows that $(K[X ; T])^{G}$ (which is identified below) is a flat $K\left[X ; T^{G}\right]$-module, and so the ring extension $K\left[X ; T^{G}\right] \subseteq$ $(K[X ; T])^{G}$ satisfies $G D(c f .[9$, Exercise 37, page 44]). In fact, this is a proper ring extension, by Proposition $2.1(b)$, since $\mathcal{O}_{G}(x)=\{x, y\}$ is a finite non-singleton set. Note that

$$
(K[X ; T])^{G}=K X^{0}+K\left(X^{x}+X^{y}\right)=\left\{0, X^{0}, X^{x}+X^{y}, X^{0}+X^{x}+X^{y}\right\}=K[u],
$$

where $u:=X^{x}+X^{y}$ satisfies $u^{2}=X^{2 x}+X^{2 y}=0$ (and $\left.X^{0}+X^{x}+X^{y}=1+u\right)$. As $(K[X ; T])^{G}$ is generated as a $K\left[X ; T^{G}\right]$-algebra by an integral element, the extension $K\left[X ; T^{G}\right] \subset(K[X ; T])^{G}$ must be integral. Finally, it is interesting to note that $(K[X ; T])^{G} \backslash K\left[X ; T^{G}\right]=\{u, 1+u\}$.

Acknowledgment. The authors would like to thank the referee for careful reading, more appropriate references. 


\section{References}

[1] M. F. Atiyah and I. G. Macdonald, Introduction to Commutative Algebra, Addison-Wesley Publishing Co., Reading, Mass.-London-Don Mills, Ont., 1969.

[2] D. E. Dobbs and J. Shapiro, Descent of minimal overrings of integrally closed domains to fixed rings, Houston J. Math., 33(1) (2007), 59-82.

[3] D. E. Dobbs and J. Shapiro, Transfer of Krull dimension, lying-over and goingdown to the fixed ring, Comm. Algebra, 35(4) (2007), 1227-1247.

[4] D. E. Dobbs and J. Shapiro, Two examples in the theory of fixed rings, Comm. Algebra, 36(3) (2008), 1097-1104.

[5] R. M. Fossum, The Divisor Class Group of a Krull Domain, Springer-Verlag, New York-Heidelberg, 1973.

[6] R. Gilmer, Multiplicative Ideal Theory, Pure and Applied Mathematics, 12, Marcel Dekker, Inc., New York, 1972.

[7] R. Gilmer, Commutative Semigroup Rings, Chicago Lectures in Mathematics, University of Chicago Press, Chicago, IL, 1984.

[8] S. Glaz, Fixed rings of coherent regular rings, Comm. Algebra, 20(9) (1992), 2635-2651.

[9] I. Kaplansky, Commutative Rings, Revised edition, The University of Chicago Press, Chicago, Ill.-London, 1974.

[10] D. G. Northcott, Topological groups and integral extensions, Quart. J. Math. Oxford Ser. (2), 32(125) (1981), 109-117.

[11] J.-P. Serre, Algèbre Locale - Multiplicités, Lecture Notes Math., 11, SpringerVerlag, Berlin, Heidelberg, New York, 1965.

\section{David E. Dobbs}

Department of Mathematics

University of Tennessee, Knoxville

Tennessee 37996-1320

e-mail: ddobbs1@utk.edu

\section{Jay Shapiro}

Department of Mathematics

George Mason University, Fairfax

Virginia 22030-4444

e-mail: jshapiro@gmu.edu 